\title{
Hierarchical Zeolites Overcome all Obstacles: Next Stop Industrial Implementation
}

\author{
Danny Verboekend, Sharon Mitchell, and Javier Pérez-Ramírez*
}

\begin{abstract}
This review emphasizes key recent accomplishments towards the industrial exploitation of hierarchically structured zeolites in catalytic processes. A major milestone comprises the demonstration that affordable post-synthetic modifications enable the transformation of any conventional zeolite into hierarchical analogues with tunable porosity and functionality. Through specific examples, belonging to the transformation of fossil fuel and renewable feedstocks, we quantitatively illustrate the spectacular benefits attained upon application of hierarchical zeolite catalysts due to improved accessibility or modification of the type and distribution of active sites. A crucial step for these exciting lab-designed materials to be implemented in industrial processes is to shape them into technical forms. Accordingly, we studied the synthesis, characterization, and catalytic evaluation of millimeter-sized hierarchical zeolite bodies, enriching the fundamental understanding on scale-up and representing an additional solid step towards the commercial application of these materials.
\end{abstract}

Keywords: Heterogeneous catalysis $\cdot$ Hierarchical zeolite $\cdot$ Post-synthetic modification $\cdot$ Scale-up

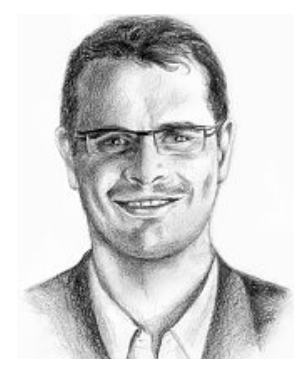

Javier PérezRamírez(Benidorm, Spain, 1974) studied Chemical Engineering at the University of Alicante, Spain and earned his $\mathrm{PhD}$ degree at TU Delft, Netherlands in 2002. After a period in the oil and fertilizer industries he was appointed ICREA research professor at ICIQ in Tarragona, Spain. In 2010, he took the chair of Catalysis Engineering at the Institute for Chemical and Bioengineering of the ETH Zurich. He is engaged in the development and understanding of new heterogeneous catalysts, multifunctional materials, and reactor engineering concepts devoted to sustainable technologies.

\footnotetext{
*Correspondence: Prof. Dr. J. Pérez-Ramírez Institute for Chemical and Bioengineering Department of Chemistry and Applied Biosciences ETH Zurich

Wolfgang-Pauli-Strasse 10

$\mathrm{CH}-8093$ Zürich

Tel.: +4144633 7120

E-mail: jpr@chem.ethz.ch
}

\section{Introduction}

Although the intrinsic properties of zeolites, e.g. strong Brønsted acidity, high surface area, high (hydro)thermal stability, and shape selectivity, make them the catalyst of choice for a plethora of reactions in various industrial sectors, they feature a major drawback in the form of access and diffusion limitations. ${ }^{[1,2]}$ Since the size of zeolite micropores (typically below $1 \mathrm{~nm}$ ) is similar to that of many molecules, transport to the active sites is dramatically slower than in larger meso- or macropores. In addition, the wide range of molecules that exceed the micropore dimensions are limited to reacting solely at the crystal's external surface. Accordingly, it has been firmly established that in most catalyzed reactions, active sites in zeolites are poorly utilized. ${ }^{[3]}$

During the last decade a persistent academic and industrial attention has focused on the development of synthetic routes to prepare more efficient zeolite catalysts. ${ }^{[4]}$ These efforts have primarily concentrated on enhancing transport in zeolite crystals either by widening the micropores, or by reducing the diffusion path length within the micropores. The latter is achieved by increasing the external surface, complementing the intrinsic zeolite micropores with a secondary level of inter- and/or intracrystalline mesopores. This family of hierarchical zeolites can be prepared using bottom-up and top-down approaches, which both prove effective in attaining superior catalytic performance compared to conventional zeolites. ${ }^{[4,5]}$
Nevertheless, the commercial viability of the majority of bottom-up approaches is hampered by the employment of costly organic templates and/or unattractive unit operations, such as centrifugal filtration and additional calcinations. ${ }^{[4]}$ On the other hand, most top-down approaches, i.e. base, acid, and steam treatments, are scalable and affordable and therefore readily implemented in an industrial context. ${ }^{[5,6]}$ In addition, each step in the post-synthetic modification protocol can be finely tuned, enabling the preparation of a wide variety of hierarchical zeolites starting from a single parent material available in the multi-ton scale. Nevertheless, independent of the synthetic approach, research on hierarchical zeolites has often been limited to laboratory studies with powders, and their true applicability remains questionable until their synthesis is upscaled to industrial manufacturing standards and they are converted into technically relevant macroscopic bodies. At this stage, these are mandatory steps to further the degree of reality of these exciting materials.

Herein we encircle the requirements for the new generation of (hierarchical) zeolite catalysts to reach industrial implementation. First we demonstrate that postsynthetic modifications represent the ideal way to prepare hierarchically-structured zeolites, being able to transform any conventional zeolite into its mesoporous analogue and, in addition, to tailor the nature and distribution of the active sites. Next, the effectiveness of these superior hierarchical zeolites is unambiguously demonstrated in several key reactions involving both estab- 


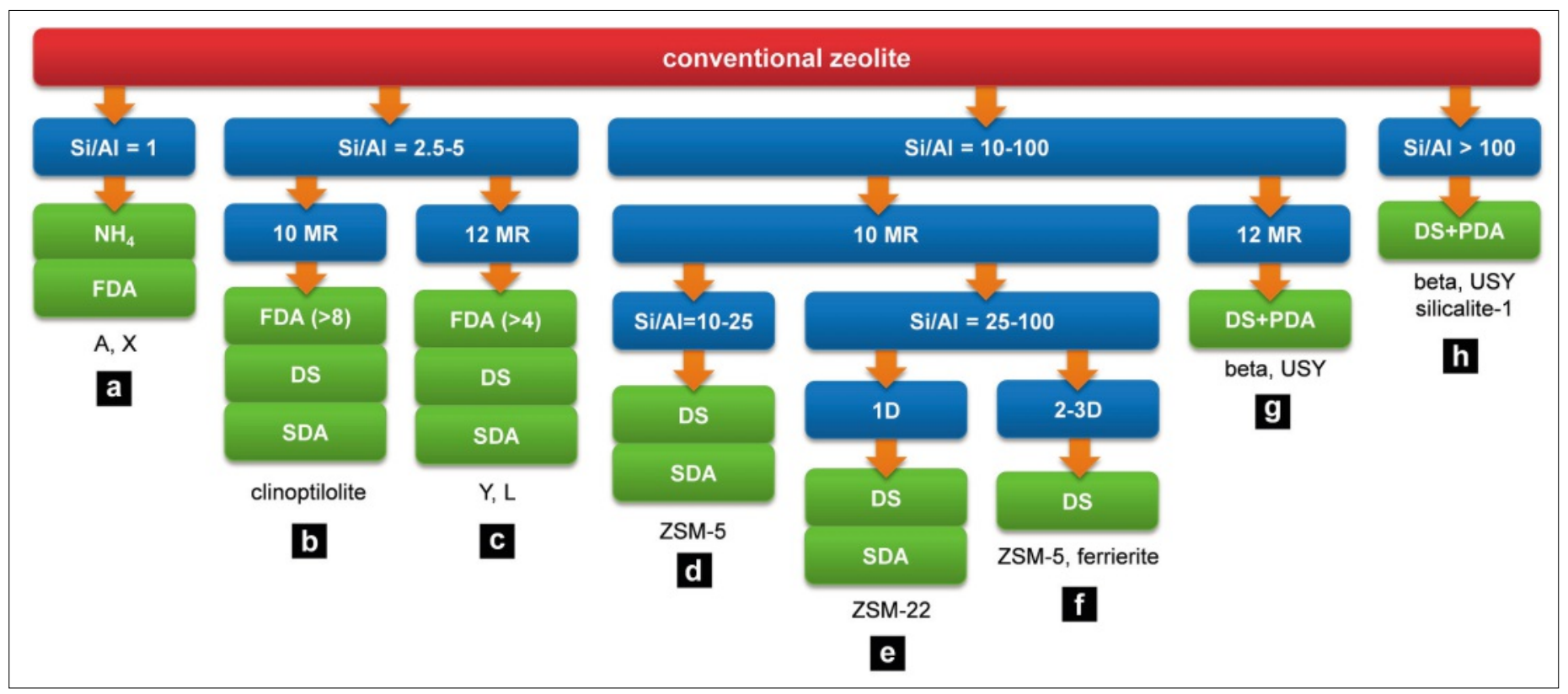

Fig. 1. Overview of post-synthetic strategies (green) to turn any conventional zeolite (red) into a hierarchical zeolite. The most important features (blue) of the conventional zeolite comprise: the Si/Al ratio, micropore size, and micropore dimensionality. Zeolites with $\mathrm{Si} / \mathrm{Al}=1 \mathrm{can}$ be brought to ammonium form $\left(\mathrm{NH}_{4}\right)$, after which a controlled framework dealumination (FDA) leads to mesopore formation (a). Parent zeolites with Si/Al = 2.5-5 can be dealuminated to facilitate subsequent dissolution by desilication (DS) using a base treatment (to $\mathrm{Si} / \mathrm{Al}>8$ and $\mathrm{Si} / \mathrm{Al}>4$, for $10-$ and $12-\mathrm{MR}$ zeolites, respectively) (b,c). For these zeolites, the removal of Al-rich debris by selective dealumination (SDA) after desilication can be of crucial importance. 10-MR zeolites in the Si/Al range 10-100 do not readily amorphize upon base leaching (d-f), but require an additional acid wash in the case the aluminum content is high (d) or when the dimensionality of the micropores is limited (e). 12-MR zeolites with Si/Al>10 require pore-directing agents (PDAs) in the alkaline solution to prevent amorphization and guide mesopore formation (g). Hierarchical high-silica zeolites (Si/Al>100) are prepared by including PDAs in the alkaline solution (h).

lished and emerging catalytic applications. Subsequently, we verify their scalability by showing their large-scale production and forming into technical catalysts bodies, while preserving their superior properties and catalytic performance. Finally, a general outlook of the field is discussed.

\section{Full Framework Flexibility}

Post-synthetic modifications, e.g. steam and acid treatments, have been applied in industry since the 1970 s to stabilize the zeolite framework and/or tune the concentration and strength of acid sites. Accordingly, as the infrastructure is present and the protocols are routinely applied, the synthesis of hierarchical zeolites by modification of conventional ones is highly attractive.

The first post-synthetic modification specifically aimed at increasing zeolite utilization by introducing a secondary network of mesopores was desilication by base leaching. This treatment was first reported by Young, ${ }^{77]}$ and later firmly established by Ogura et al..$^{[8]}$ and Groen et al. ${ }^{[9]}$ Initially, desilication was reported to be most efficient for ZSM-5 zeolites in the compositional $\mathrm{Si} / \mathrm{Al}$ range $25-50$, where framework aluminum was coined 'poredirecting agent' due to its regulatory effect on mesopore formation. This major limitation sparked the development of a wide array of post-synthetic modification strategies, which have enabled the full topological and compositional flexibility. ${ }^{[10-12]}$ In addition, their carefully orchestrated application permits the tailoring of other zeolite characteristics, e.g. the composition and functionality. ${ }^{[13]}$ In Fig. 1 the distinct postsynthetic strategies are organized based on a number of key properties of the parent zeolites. In order of importance, these criteria comprise the $\mathrm{Si} / \mathrm{Al}$ ratio $(1-\infty)$, the micropore size (10-12-membered ring, $\mathrm{MR}$ ), and the micropore dimensionality (1D-3D). The post-synthetic sequences include four different treatments:

Desilication (DS): The mesopores formed by desilication originate from a controlled (ideally intracrystalline) leaching of the zeolite framework using alkaline aqueous solutions. Although both $\mathrm{Al}$ and $\mathrm{Si}$ species are removed from the framework, the extracted aluminum is often reincorporated in the zeolite, where it acts as a pore-directing agent. Base treatment using aqueous $\mathrm{NaOH}$ (in the absence of external pore-directing agents, vide infra) is suited for $10-\mathrm{MR}$ zeolites with $8<\mathrm{Si} / \mathrm{Al}<100$ (b, d-f), and for $12-\mathrm{MR}$ zeolites $4<\mathrm{Si} / \mathrm{Al}<10$ (c). Alkaline treatments require optimization, primarily in terms of alkalinity, depending among others factors on the morphology, $\mathrm{Si} / \mathrm{Al}$ ratio, and micropore size. For example, ZSM-5 (10-MR, Si/Al = 15) requires relatively severe conditions (e.g. $>0.6 \mathrm{M} \mathrm{NaOH}$ ), while 12-MR zeolites with similar composition necessitate only a mild alkalinity (e.g. USY: $0.1 \mathrm{M} \mathrm{NaOH}$ ) to induce dissolution and mesopore formation.

Desilication with external pore-directing agents $(D S+P D A)$ : The use of inorganic (soluble $\mathrm{Al}$ and $\mathrm{Ga}$ salts) and organic additives (tetraalkylammonium cations, TAAs) as additives to the alkaline solutions proved of high value. Because of their specific interaction with the zeolite surface under the treatment conditions they influence the degree and mechanism of dissolution, which can be exploited to tune both the mesopore size and the surface acidity. ${ }^{[13]}$ Moreover, in addition to framework $\mathrm{Al}$, they exert a pore-directing role, able to direct the leaching process even within high-silica zeolites ( $\mathrm{Si} / \mathrm{Al}>100$, h). Finally, the addition of organic PDAs, especially TAAs, in the alkaline solution prevents framework amorphization, which is crucial in the preparation of hierarchical 12-MR zeolites with $\mathrm{Si} / \mathrm{Al}>10$ (g).

Selective dealumination (SDA): A prominent feature of base treatment is that when aluminum is reincorporated in the solid, it can thwart the intrinsic zeolitic properties. Especially in zeolites of low $\mathrm{Si} / \mathrm{Al}$ ratio $(<25, \mathbf{b}-\mathbf{d})$ and unidirectional micropores (e), this 'realumination' process can cause extensive blockage of micro- and mesopores. By using a subsequent mild acid wash, n.b. not aimed at the removal of aluminum in lattice positions, 
the deposited species can be dissolved, yielding a hierarchical zeolite with a $\mathrm{Si}$ / Al ratio, crystallinity, microporosity, and acidity nearer to that of the parent sample.

Framework dealumination (FDA): Mesopore formation is not always possible by direct base treatment, due to the very slow dissolution rate of Al-rich zeolites. In this case, one option is to increase the $\mathrm{Si}$ / Al ratio to enable subsequent dissolution by base treatment. The $\mathrm{Si} / \mathrm{Al}$ ratio which needed to be reached to permit efficient mesopore formation was found to depend on the characteristic ring size of the zeolite framework, being $c a$. 8 in the case of 10-MRs (b) and 4 for 12-MRs zeolites (c). The efficiency of the initial dealumination strongly influences the impact of the subsequent alkaline treatment and the requirement for a final acid wash. ${ }^{[10]}$ Importantly, the optimized dealumination of the framework by a controlled acid leaching can also lead to extensive mesoporosity. For example, acid treatment using EDTA complexes enabled the development of mesopores in Al-rich zeolites such as $\mathrm{Y}(\mathrm{Si} / \mathrm{Al}=2.4)$ and $\mathrm{X}$ and $\mathrm{A}(\mathrm{Si} / \mathrm{Al}=1.2)(\mathbf{a})$. In the latter cases, the type of charge-balancing cation present in the zeolite was key to achieving extensive mesopore formation.

\section{Traditional Catalytic Applications}

The most critical objective in the design of any hierarchical zeolite is to attain superior performance in specific catalyzed processes. Hierarchical zeolites have proved their superiority to conventional zeolites in a wide variety of reactions. ${ }^{[14]}$ Here we illustrate the benefits of hierarchical zeolites prepared by post-synthetic design in several major petrochemical catalytic reactions involving either coupling, cracking, or isomerization of hydrocarbons and attaining improved activity, selectivity, and/ or stability.

Hierarchical zeolite catalysts are frequently evaluated in acid-catalyzed $\mathrm{C}-\mathrm{C}$ bond formations such as alkylations. In the liquid phase, these couplings mostly occur on the external surface of the crystals, ${ }^{[15]}$ and are accordingly excellent model reactions highlighting the potential of designing hierarchical zeolites using acid and base treatments. Hierarchical L, ${ }^{[10]} \mathrm{Y},{ }^{[11]}$ and ZSM-5 ${ }^{[15]}$ zeolites prepared using the protocols in Fig. 1, have demonstrated that, while the selectivity is maintained, impressive activity gains up to an order of magnitude can be obtained. Based on the enhanced access, alternative zeolite frameworks, which have so far not been able to compete, may become suitable for previously unthinkable applications. For example, it was recently demonstrated that the natural zeolite clinoptilolite, normally poorly active, achieved a similar performance to the commonly-applied ZSM-5 in the alkylation of toluene with benzyl alcohol, after it was brought to the hierarchical form by post-synthetic modifications. ${ }^{[10]}$ In addition, we anticipate that hierarchical zeolites will also be viable catalysts for reactions that were hitherto performed using mineral acids like $\mathrm{HCl}$, e.g. hydroxyalkylations. ${ }^{[16]}$

Alternatively, an important route to prepare complex hydrocarbons is the conversion of methanol, commonly derived from coal or natural gas, into olefin or gasoline-range products, i.e. MTO or MTG. ${ }^{[1,2]}$ While zeolite catalysts based on ZSM-5 or SAPO-34 are selective for these processes, they suffer from rapid deactivation due to coking, meaning that catalyst stability is a key performance descriptor. Also in this reaction, post-synthetically modified hierarchical zeolites exhibit superior performance, displaying lifetimes up to $c a$. fourfold that of the conventional zeolite, while maintaining similar selectivity patterns. ${ }^{[5,17,18]}$

The largest application of zeolite catalysts by volume (ca. $95 \mathrm{vol} . \%$ ) is the fluid catalytic cracking of vacuum gas oil (VGO).[1,2,19] This process, involving roughly $10 \%$ of each barrel of oil, traditionally uses steamed, acid leached, and rare-earth exchanged $Y$ zeolites to convert heavy fractions of crude oil into lighter and more valuable gasoline and diesel fractions. ${ }^{[19]}$ Using the strategy applied in Fig. $1 \mathrm{c}, \mathrm{Y}$ zeolites can be prepared combining a preserved crystallinity and microporosity with an intracrystalline secondary pore system (Fig. 2a,b). After stabilization of these zeolites by steaming and/or lanthanum ion exchange, similar activities were attained. Taking into account that the hierarchical zeolite possessed only $\sim 20 \%$ of the acidity of the parent zeolite (Table 1), this implies a higher utilization of the remaining acid sites. More importantly, a higher diesel yield was obtained at the expense of undesirable light gas formation (Fig. 2d,e). ${ }^{[20]}$

Another key catalytic process in the petrochemical industry is the skeletal hydro-isomerization of long alkanes to improve their rheological properties at low temperature. ${ }^{[1,2,19]}$ This process, also referred to as dewaxing, makes use of bi-
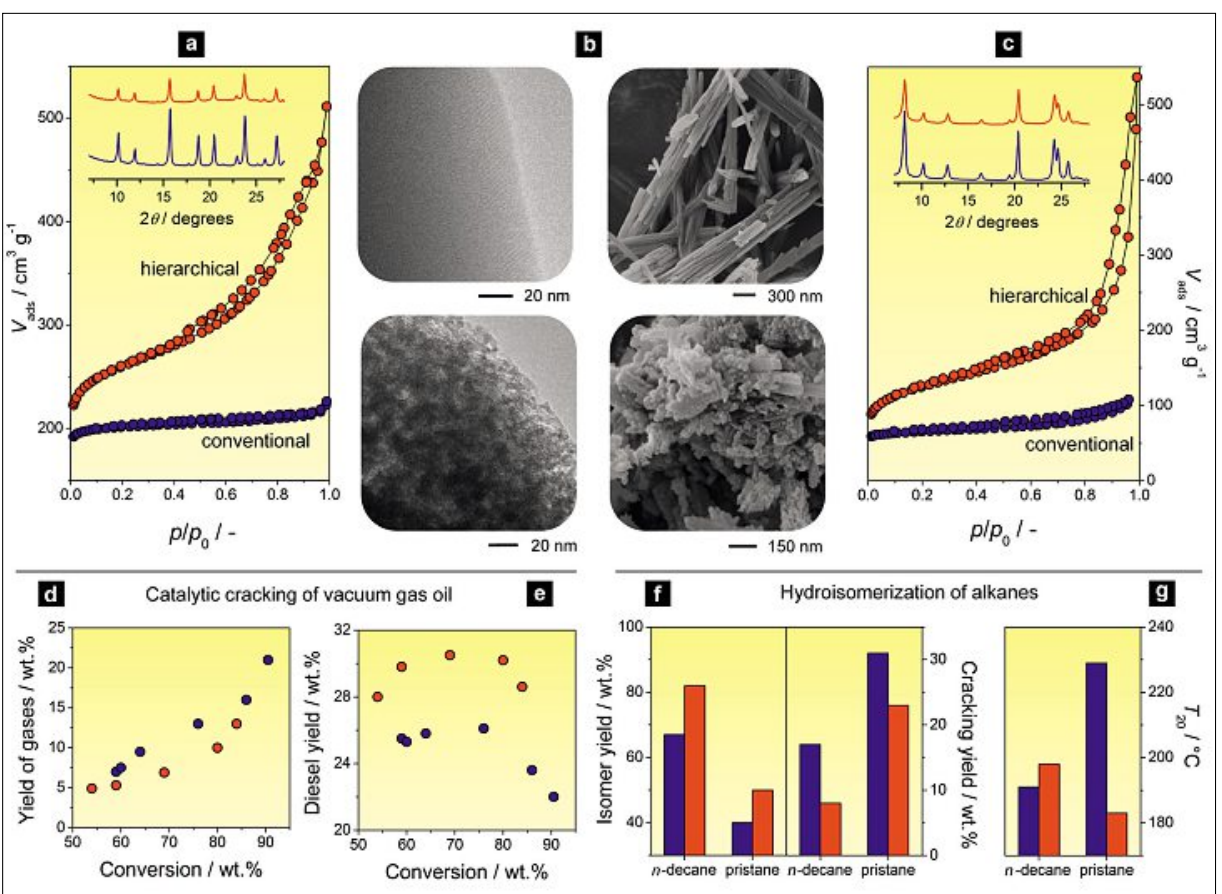

Fig. 2. Characterization and catalytic evaluation of conventional (blue) and hierarchical (red) $Y$ and ZSM-22 zeolites. (a,c) Nitrogen isotherms of conventional and hierarchical $Y$ and ZSM-22 zeolites, respectively. The enhanced uptake at $p / p_{0}>0.1$ indicates the presence of substantial mesoporosity. Insets: X-ray diffraction patterns confirming the preservation of the long-range crystallinity. (b) TEM and SEM images of conventional (top) and hierarchical (bottom) Y (left) and ZSM-22 (right) zeolites, respectively. Catalytic evaluation of conventional and hierarchical $Y$ zeolites in the cracking of vacuum gas oil (d,e). The hierarchical sample yields less (d) undesired gases and (e) more diesel. Catalytic evaluation of conventional and hierarchical ZSM-22 in the hydroisomerization of $n$-decane and pristine $(\mathbf{f}, \mathbf{g})$. The hierarchical sample displays a higher selectivity to isomers at the expense of cracking (f).With larger or more branched substrates, e.g. pristane, the hierarchical zeolite additionally displays a higher activity, hence lower temperature at $20 \%$ conversion $\left(T_{20}, \mathbf{g}\right)$. The enhanced catalytic performances are particularly striking taking into account that the hierarchical samples display strongly reduced acidities compared to the conventional zeolites (Table 1). These results emphasize the pronounced selectivity benefits that can be obtained by tailoring the accessibility, nature, and distribution of the active sites in zeolites. 
Table 1. Acidity of conventional and hierarchical $Y^{[20]}$ and ZSM-22 ${ }^{[22]}$ zeolites determined by infrared spectroscopy of adsorbed pyridine.

\begin{tabular}{|c|c|c|}
\hline Zeolite & $\begin{array}{l}\text { Brønsted } \\
\text { acid sites } \\
\mu \mathrm{mol} \mathrm{g}{ }^{-1}\end{array}$ & $\begin{array}{l}\text { Lewis } \\
\text { acid sites } \\
\text { بmol g-1 }\end{array}$ \\
\hline Y conventional & 204 & 89 \\
\hline Y hierarchical & 28 & 36 \\
\hline $\begin{array}{l}\text { ZSM-22 } \\
\text { conventional }\end{array}$ & 208 & 35 \\
\hline $\begin{array}{l}\text { ZSM-22 } \\
\text { hierarchical }\end{array}$ & 113 & 48 \\
\hline
\end{tabular}

functional 10-MR zeolite catalysts, e.g. ZSM-22 and ferrierite, containing a minute amount of noble metal. Such zeolite catalysts display exceptional isomer selectivities, due to a so-called pore mouth keylock mechanism. ${ }^{[21]}$ Hierarchical ZSM-22 zeolites were prepared using the strategy indicated in Fig. 1e, displaying a high crystallinity and microporosity. In addition, the fragmented needle-like crystals led to a large external surface (Fig. 2b,c), hereby providing a higher density of pore mouths and shorter diffusion pathways through the unidirectional ZSM-22 micropores. As a result, increased yields of the desired isomers were attained at the expense of light gas formation (Fig. 2f). The latter was, as in the case of the catalytic cracking of VGO, attributed to the reduced (over) cracking caused by the shortened micropore diffusion pathlength in combination with the milder acidity. ${ }^{[21]}$ This clearly demonstrated the fine interplay between the accessibility, nature, and abundance of the acid sites, and highlights that, besides activity and stability benefits, hierarchical zeolites are capable of attaining pronounced selectivity improvements. In fact, based on the enhanced active site accessibility, many structure-property-function relationships established over the last several decades for conventional zeolites may not be applicable for hierarchical zeolites. This suggests that, with secondary porosity established as an important descriptor, zeolite design needs to be re-evaluated as a whole.

\section{Emerging Catalytic Applications}

Hierarchical zeolites prepared by postsynthetic design not only excel in classical petrochemical conversions. The preservation of the intrinsic zeolitic properties combined with the enhanced external surface and the option to tune the distribu-

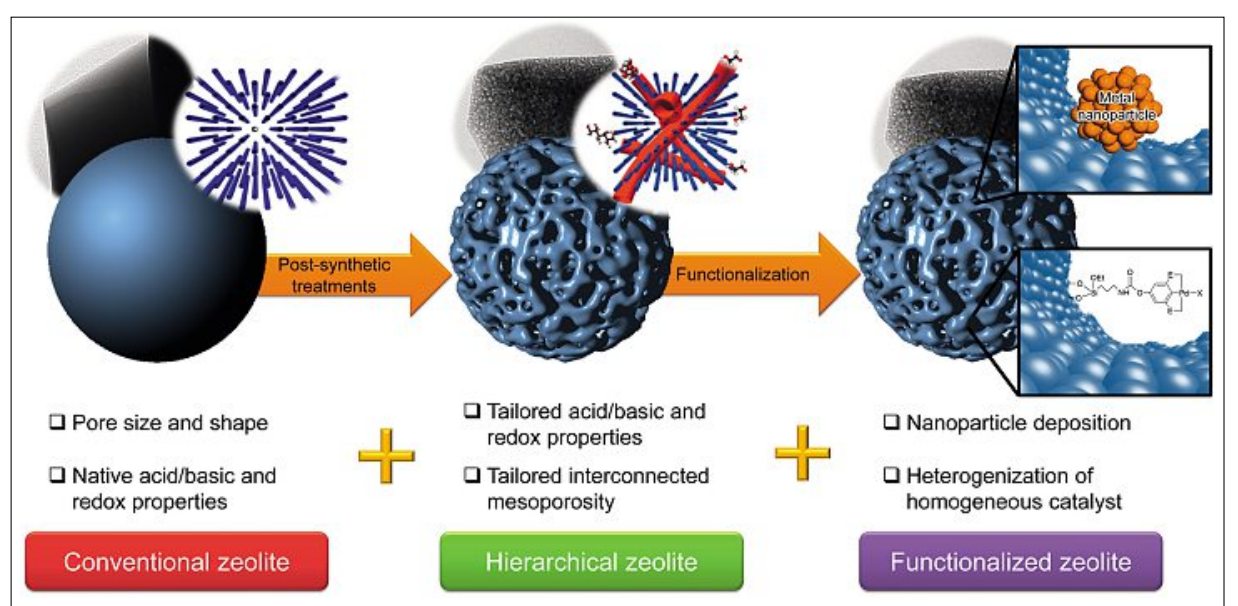

Fig. 3. Design aspects of zeolites at different stages in the transformation of a conventional microporous zeolite into a superior (functionalized) mesoporous zeolite. Based on the enhanced secondary porosity the acid/base/redox requirements in catalytic applications determined for conventional and hierarchical zeolites differ dramatically. tion and nature of the active sites, opens a window of opportunity in several emerging applications (Fig. 3). For example, in the case of hydro-isomerization using ZSM22 zeolite it was found that, while exhibiting a lower total acidity than the parent zeolite (Table 1), activity gains were observed over the hierarchical zeolite when applied for the conversion of longer and more branched molecules, e.g. pristane (Fig. 2g). ${ }^{[22]}$ While this of course further stresses the importance of accessibility in hierarchical zeolite catalysts, it also makes the hierarchical Pt/ZSM-22 particularly suited for processing renewable hydrocarbon sources. An example hereof is the longer alkanes produced in Fischer-Tropsch catalysis, and the highly branched ones derived from algae processing, as in the case of pristane. ${ }^{[22]}$

The use of zeolites in the conversion of biomass has come into the spotlight more recently. ${ }^{[23]}$ For example, in the isomerization of dihydroxyacetone to lactic acid, an important chemical intermediate, the use of alkaline-treated ZSM-5 zeolites originated a fourfold increase of the selectivity to lactic acid (Fig. 4a,b). [24] Strikingly, an in-depth characterization revealed that the enhanced selectivity did not originate from the introduced mesoporosity, but from the presence of Lewis acid sites formed upon base treatment. This was confirmed by the relatively poor performance of a highly mesoporous zeolite from which the aluminum species were subsequently removed by selective dealumination. This first-time demonstration of the successful exploitation of the 'alkali-induced realumination' on the external surface highlights the tremendous flexibility that the post-synthetic toolbox offers, not only to alleviate diffusion limitations but also to create specific acid sites. Unfortunately, the increasing $\mathrm{pH}$ of the aqueous solution upon formation of lactic acid, led to the partial elimination of these highly selective sites. Accordingly, a reduced performance was obtained upon re-using of the catalyst in sequential runs, identifying their stabilization (in aqueous solutions) as a major challenge.

In heterogeneous catalysis, the role of solid bases is less pronounced than that of solid acid catalysts. Moreover, rather than

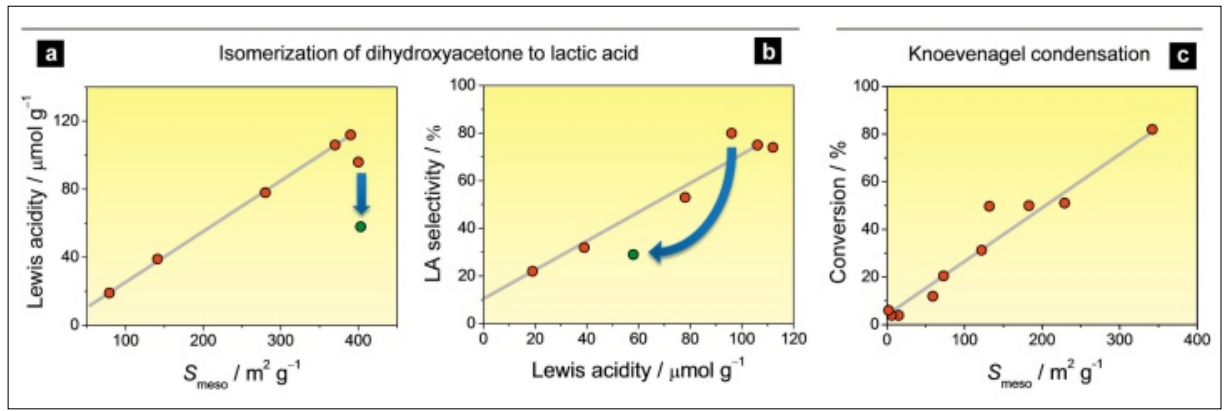

Fig. 4. Concentration of Lewis acid sites versus the mesopore surface area $\left(S_{\text {meso }}\right)$ of alkalinetreated ZSM- 5 zeolites (a). Lactic acid (LA) selectivity in the isomerization of dihydroxyacetone as a function of Lewis acidity (b). The effect of the sequential acid washing of a highly mesoporous sample is indicated by the arrow and green symbol in $(\mathbf{a}, \mathbf{b})$. The conversion of benzaldehyde in the Knoevenagel condensation of benzaldehyde with malononitrile as a function of the $S_{\text {meso }}$ for various Cs-exchanged and nitrided FAU and LTA-type zeolites (c). 
zeolites, metal oxides and hydrotalcite-derived materials are predominantly used in existing applications. ${ }^{[25]}$ The latter is attributed to the fact that zeolites only possess weak Lewis basicity and because relevant reactions often involve substrates larger than the zeolite micropores ( $>1 \mathrm{~nm})$. In an attempt to probe access and diffusion limitations in these types of reactions, hierarchical zeolites were transformed into base catalysts by alkali-ion exchange and nitridation. ${ }^{[12]}$ The resulting materials, entirely prepared by post-synthetic design, were tested in the Knoevenagel condensation of benzaldehyde with malononitrile, where they displayed remarkable activities of up to 10 times that of the conventional zeolite. ${ }^{[12]}$ In this case, the reaction was found to mainly take place on the external surface, and that the contribution of the latter descriptor largely outweighed that of the total basicity (Fig. 4c).

\section{Successful Scale-up}

The scale-up of catalyst powders into technically-relevant forms represents a critical phase in catalyst development, for which little fundamental knowledge has been generated in the public arena. ${ }^{[26]}$ The lack of academic interest in this topic could be down to a number of reasons. Perhaps the most upfront explanation is that small-scale catalytic testing involves typically only several grams of catalysts, and, moreover, does not require the active phase to be part of a mechanically strong and attrition-resistant shape. As a result, pressing criteria and key descriptors for the purpose-design of technical catalysts remain difficult to identify. Next, the infrastructure required to (i) scale-up the synthesis of the different components, (ii) to representatively shape powders, and (iii) to characterize the resulting bodies, may require substantial investment. Nevertheless, by combining novel synthesis and characterization techniques with smart experimentation and collaboration with industrial partners, our group has set out to prove the superiority of hierarchical zeolite in technical form, hereby gathering fundamental understanding on the scale-up and shaping of heterogeneous catalysts in general.

After the first tentative efforts of Groen et al., ${ }^{[27]}$ a number of crucial achievements towards the preparation of hierarchical zeolites in technical form have been recently achieved. First of all, we successfully prepared $\mathrm{mm}$-sized hierarchically-structured zeolite catalysts by demonstrating that (i) the post-synthetic modification of a ZSM-5 zeolite according to the treatment strategy shown in Fig. 1f was reproducible upon translation from the gram (Fig. 5a) to

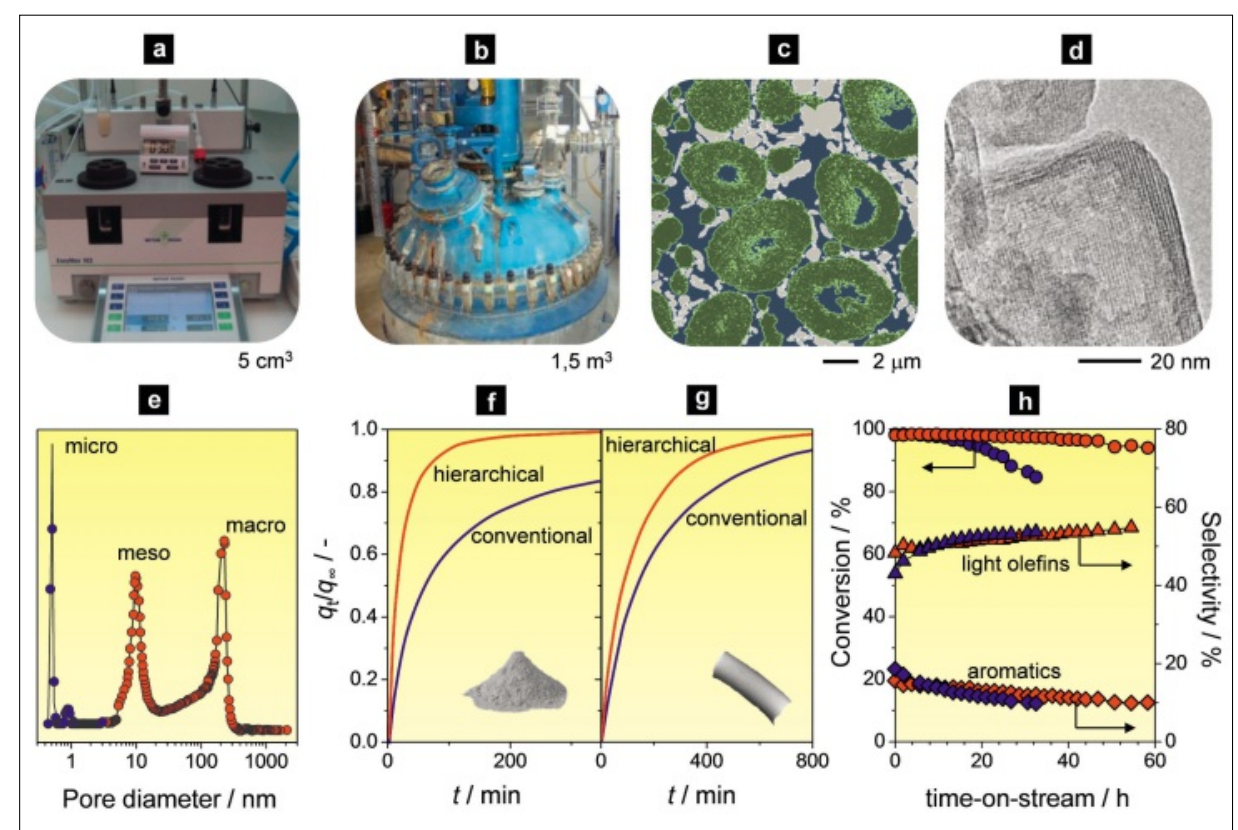

Fig. 5. Achievements in the scale-up and shaping of conventional (blue) and hierarchical (red) zeolites. Successful extrapolation of the preparation of hierarchical zeolite powders from lab tubes of $5 \mathrm{~cm}^{3}$ (a) to a pilot-scale reactor of $1.5 \mathrm{~m}^{3}(\mathbf{b})$. Visualization of the multidimensional pore network within hierarchical zeolite bodies using, among many other state-of-the-art microscopic methods, focused ion beam- (FIB-) SEM (c) and TEM (d). Characterization of the different porosity levels in hierarchical zeolite bodies using $\mathrm{Ar}$ and $\mathrm{N}_{2}$ adsorption in combination with $\mathrm{Hg}$ intrusion, obtaining pore size distributions from the nanometer to the micrometer range (e). Increased diffusivity observed over powder samples $(\mathbf{f})$ is preserved after shaping into extrudates $(\mathbf{g})$. Extended life time in the conversion of methanol to olefins using hierarchical zeolite extrudates (h).

the kilogram scale (Fig. 5b), ${ }^{[6]}$ and (ii) that the hierarchical porosity was preserved upon agglomeration of the mesoporous ZSM-5 powders attained with different natural clay binders into granules and extrudates. ${ }^{[6,28]}$ Detailed characterization of these shaped forms was undertaken to understand the precise impact of the shaping process on the properties of the hierarchical zeolites. For example, numerous state-of-the-art imaging techniques were employed to examine the internal structure from the macro- to the nanoscale (Fig. 5c,d). The insights gained into the various porosity levels of the shaped bodies greatly complemented those obtained from other conventional characterization techniques, i.e. gas adsorption and $\mathrm{Hg}$ porosimetry (Fig. 5e). ${ }^{[18]}$

Beneficial impacts observed on application of hierarchical zeolites are commonly attributed to an enhanced mass transfer, without direct verification of the latter. Through gravimetric study of the adsorption of 2,2-dimethylbutane, a hydrocarbon probe of similar kinetic diameter to the dimensions of the MFI micropores, we recently evidenced an increased diffusivity over hierarchical ZSM-5 zeolites compared with their conventional microporous counterpart (Fig. 5f). Advantageously in relation to their scale-up, faster uptakes were also retained in hierarchical zeolite extrudates (Fig. 5g), [29] indicating that the incorporation of the binder and the mechanical steps of shaping did not adversely impact the enhancing role of the intracrystalline mesopores, the latter being of critical relevance to those working on hierarchical zeolites in general. Finally, the greatly extended lifetime displayed over the modified mesoporous zeolites in the conversion of methanol to olefins confirmed their superior performance in technical form (Fig. 5h). ${ }^{[18]}$

\section{Conclusions and Outlook}

The tremendous progress in the design, application, and scale-up of hierarchical zeolites prepared by post-synthetic modifications boost the prospects of their shortterm industrial implementation. However, several challenges remain, providing substance for numerous exciting academic and industrial studies to come.

For example, some fundamental issues concerning the mesopore formation by alkaline and acid treatments have not been fully addressed. Although general rules and guidelines were devised over the last decade, the understanding of mesopore formation at a molecular level is still lacking. Both techniques with sufficient spatial and time resolution, as well as theoretical studies could shed more light on this. In addition, while they were already exploited in catalyzed reactions (Fig. 4a,b), the exact nature of the aluminum sites covering the 
external surface (i.e. the realuminated species) has not been conclusively identified.

With the post-synthetic tools developed, application-oriented design is anticipated, involving the re-evaluation of all key descriptors. Such a tailored catalyst development should involve precise preparation and extended catalytic testing. Besides the established academic interest, we expect that the readily available and easily implemented post-synthetic toolbox will be eagerly absorbed by leading companies, tailoring hierarchical zeolites to the smallest detail to their specific on-site demands.

Knowing that any zeolite can be brought into hierarchical form, an increased application of hierarchical zeolites is envisaged. For example, the potential of hierarchical zeolites as improved ion exchangers and adsorbents may constitute a novel avenue of research. In addition, the enhanced mesopore surface area also provides a chance to utilize the unique characteristics of zeolites in novel applications (Fig. 3). For example, the use of a hierarchical zeolite as a carrier for a metal phase is expected to be favorable for obtaining a superior metal dispersion, while a similar approach could be envisaged to tether homogenous moieties. Ideally, the deposited species should bring an additional functionality to allow hierarchical conversions to take place, e.g. a first conversion in the micropore, followed by a secondary conversion on the mesopore surface.

While demonstrating proof-of-concept, the promising results on the scale-up and shaping have, so far, been limited to one zeolite framework-type and a single catalytic application. The scalability of other treatment strategies illustrated in Fig. 1 remains to be proved and the impact of shaping on the properties of other hierarchical zeolites obtained has yet to be established. For example, we expect that hierarchical USY zeolites, being less stable than hierarchical ZSM-5, should pose an even larger challenge to scale-up.

As discussed, the post-synthetic modification of hierarchical zeolite powders can be considered as a tool to tune not only the porous properties, but also the type and distribution of active sites present. Herein, the appropriate choice of binders and other additives applied during scale-up in relation to their impact on the porosity of the shaped bodies, and the introduction of chemically-distinct functionality, needs to be rationalized with respect to the optimal properties of the zeolite phase. As this differs for different chemical processes, the performance of hierarchical zeolites in technical form needs to be evaluated in a broad range of reactions.

Finally, critical economic analyses of the preparation of the hierarchical zeolite powder, the shaping process, and the benefits in the catalyzed reactions should be carried out. For example, in the case of post-synthetic acid or base leaching, it is important to evaluate whether the reported benefits in catalysis outweigh the costs of solids lost by dissolution and the subsequent additional wastewater treatment. Of course, to make these economic analyses in an accurate fashion, a tight collaboration with industrial partners is of crucial importance.

\section{Acknowledgement}

The Swiss National Science Foundation (Project Numbers 200021-134572 and 200021140496) is acknowledged.

Received: March 20, 2013

[1] A. Corma, Chem. Rev. 1995, 95, 559.

[2] W. Vermeiren, J.-P. Gilson, Top. Catal. 2009 , 52, 1131 .

[3] J. Pérez-Ramírez, C. H. Christensen, K. Egeblad, C. H. Christensen, J. C. Groen, Chem. Soc. Rev. 2008, 37, 2530.

[4] a) R. Chal, C. Gérardin, M. Bulut, S. van Donk, ChemCatChem 2011, 3, 67; b) S. LopezOrozco, A. Inayat, A. Schwab, T. Selvam, W. Schwieger, Adv. Mater. 2011, 23, 2602; c) J. Pérez-Ramírez, Nat. Chem. 2012, 4, 250; d) K. Egeblad, C. H. Christensen, M. Kustova, C. H. Christensen, Chem. Mater. 2008, 20, 946.

[5] D. Verboekend, J. Pérez-Ramírez, Catal. Sci. Technol. 2011, 1, 879.

[6] J. Pérez-Ramírez, S. Mitchell, D. Verboekend, M. Milina, N.-L. Michels, F. Krumeich, N. Marti, M. Erdmann, ChemCatChem 2011, 3, 1731.

[7] D. A. Young, US Patent, 3326797, 1967.

[8] M. Ogura, S.-Y. Shinomiya, J. Tateno, Y. Nara, E. Kikuchi, M. Matsukata, Chem. Lett. 2000, 882 .

[9] J. C. Groen, L. A. A. Peffer, J. A. Moulijn, J. Pérez-Ramírez, Chem. Eur. J. 2005, 11, 4983.
[10] D. Verboekend, T. C. Keller, M. Milina, R. Hauert, J. Pérez-Ramírez, Chem. Mater. 2013, DOI: $10.1021 / \mathrm{cm} 4006103$.

[11] a) D. Verboekend, G. Vilé, J. Pérez-Ramírez, Adv. Funct. Mater. 2012, 22, 916; b) D. Verboekend, J. Pérez-Ramírez, Chem. Eur. J. 2011, 17, 1137; c) D. Verboekend, A. M. Chabaneix, K. Thomas, J. -P. Gilson, J. PérezRamírez, CrystEngComm 2011, 13, 3408; d) D. Verboekend, S. Mitchell, M. Milina, J. C. Groen, J. Pérez-Ramírez, J. Phys. Chem. C 2011, 115, 14193.

[12] D. Verboekend, T. C. Keller, S. Mitchell, J. Pérez-Ramírez, Adv. Funct. Mater. 2013, DOI: 10.1002/adfm.201202320.

[13] a) J. Pérez-Ramírez, D. Verboekend, A. Bonilla, S. Abelló, Adv. Funct. Mater. 2009, 19, 972; b) D. Verboekend, G. Vilé, J. Pérez-Ramírez, Cryst. Growth Des. 2012, 12, 3123.

[14] M. S. Holm, E. Taarning, K. Egeblad, C. H. Christensen, Catal. Today 2011, 168, 3.

[15] M. Milina, S. Mitchell, Z. Domínguez Trinidad, D. Verboekend, J. Pérez-Ramírez, Catal. Sci. Technol. 2012, 2, 759.

[16] A. de Angelis, P. Ingallina, C. Perego, Ind. Eng. Chem. Res. 2004, 43, 1169.

[17] S. Mitchell, N.-L. Michels, K. Kunze, J. PérezRamírez, Nat. Chem. 2012, 4, 825.

[18] S. Svelle, L. Sommer, K. Barbera, P. N. R. Vennestrom, U. Olsbye, K. P. Lillerud, S. Bordiga, Y.-H. Pan, P. Beato, Catal. Today 2011, 168, 38 .

[19] G. Alfk W. W. Irion, O. S. Neuwirth, 'Oil Refining', in 'Ullmann's Encyclopedia of Industrial Chemistry', Wiley, Weinheim, 2012.

[20] C. Martínez, D. Verboekend, J. Pérez-Ramírez, A. Corma, Catal. Sci. Technol. 2013, 3, 972.

[21] J. A. Martens, W. Souverijns, W. Verrelst, R. Parton, G. F. Froment, P. A. Jacobs, Angew. Chem. Int. Ed. 1995, 34, 2528.

[22] J. A. Martens, D. Verboekend, K. Thomas, G. Vanbutsele, J.-P. Gilson, J. Pérez-Ramírez, ChemSusChem 2013, 6, 421.

[23] P. Y. Dapsens, C. Mondelli, J. Pérez-Ramírez, ACS Catal. 2012, 2, 1487.

[24] P. Y. Dapsens, C. Mondelli, J. Pérez-Ramírez, ChemSusChem, 2013, DOI:10.1002/ cssc. 201200703.

[25] a) Y. Ono, J. Catal., 2003, 216, 406; b) H. Hattori, Chem. Rev. 1995, 95, 537.

[26] a) A. B. Stiles, T. A. Koch, 'Catalyst Manufacture', Marcel Dekker Inc., New York 1995; b) F. Schüth, M. Hesse, in 'Handbook of Heterogeneous Catalysis', Vol. 1, Eds: G. Ertl, H. Knözinger, J. Weitkamp, Wiley-VCH, Weinheim, Germany 2008, Ch. 2.

[27] J. C. Groen, J. A. Moulijn, J. Pérez-Ramírez, Ind. Eng. Chem. Res. 2007, 46, 4193.

[28] N. -L. Michels, S. Mitchell, M. Milina, K. Kunze, F. Krumeich, F. Marone, M. Erdmann, N. Marti, J. Pérez-Ramírez, Adv. Funct. Mater. 2012, 22, 2509.

[29] L. Gueudré, M. Milina, S. Mitchell, J. PérezRamírez, Adv. Funct. Mater. 2013, DOI: 10.1002/adfm.201203557. 